\title{
Frankincense-derived triterpenoid improves psoriasis
}

The finding that a natural triterpenoid that inhibits nuclear factor $\kappa \mathrm{B}(\mathrm{NF} \kappa \mathrm{B})$ signaling "can reverse the disease state in a mouse model of psoriasis, in terms of clinical, biochemical and microscopical parameters...might pave the road to an NFkB-targeted treatment of this chronic inflammatory disease," claims Thomas Simmet, of the Institute of Pharmacology of Natural Products and Clinical Pharmacology, at Ulm University in Germany.

\section{4 ...mice with a severe inflammatory phenotype... showed a considerable improvement... 77}

Simmet's group had previously sought to identify how triterpenoids of the boswellic acid family isolated from oleogum resins of Boswellia species might impart the therapeutic effect conferred by this traditional medicine-more commonly known as frankincense-on various inflammatory diseases. Acetyl-11-keto- $\beta$-boswellic acid (AK $\beta$ BA) directly inhibited the inhibitor of NFkB kinase complex. Subsequent inhibition of the NFkB pathway suppressed the induction of various NFkB-dependent proinflammatory cytokines, such as tumor necrosis factor (TNF). In vivo, systemic administration of $\mathrm{AK} \beta \mathrm{BA}$ downregulated the expression of several NFKB targets, including chemokines and cytokines, leading to therapeutic efficacy in an established model of mouse atherosclerosis.

A number of NFkB-induced cytokines, including TNF, several interleukins and interferon- $\alpha$ and interferon- $\beta$, are involved in the pathogenesis of psoriasis, prompting Simmet's group to investigate the effect of AK $\beta B A$ on the CD18 hypomorphic $\left(\mathrm{CD} 18^{\text {hypo }}\right)$ mouse model of psoriasis in the present study. First, though, the researchers established that NFkB was activated in skin biopsies from psoriatic lesions of these mice compared with those from normal mice. Psoriasis has historically been viewed as a T-cell-mediated disease, but macrophages are increasingly becoming recognized as a potential source of proinflammatory mediators; indeed, activated macrophages are the main source of TNF in the CD18 $8^{\text {hypo }}$ model. TNF expression is induced by, and potently activates, NFkB signaling, and Simmet's group found a massive increase in the number of macrophages with activated $\mathrm{NF \kappa B}$ in the dermis of human psoriatic skin compared with normal skin. Similarly, macrophages in psoriatic lesions from CD18 $8^{\text {hypo }}$ mice showed NFkB activation, and were identified as the main source of TNF in this model.

Returning to the issue of the effect of AK $\beta B A$ on $C D 18^{\text {hypo }}$ mice, treatment of the animals with either $30 \mu \mathrm{mol} / \mathrm{kg}$ or
$100 \mu \mathrm{mol} / \mathrm{kg}$ of the natural compound improved inflammation after 35 days. Notably, mice with a severe inflammatory phenotype treated with $100 \mu \mathrm{mol} / \mathrm{kg}$ showed a considerable improvement in symptoms. NFKB activation was also inhibited, and levels of TNF, interleukin (IL)-12, IL-13 and monocyte chemoattractant protein-1 were reduced. AK $\beta B A$ also inhibited keratinocyte proliferation, but did not affect NFkB signaling in these cells, owing to the expression of a multidrug resistance-associated protein.

Finally, the researchers selectively targeted AK $\beta \mathrm{BA}$ to skin macrophages in CD18 ${ }^{\text {hypo }}$ mice using liposomes, leading to a remarkable improvement of the skin inflammation after 3 weeks in animals with a very severe phenotype.

Not only does this compound hold promise for the treatment of psoriasis, but it might also be effective against a variety of other chronic inflammatory diseases. "The compound should be further developed for topical application, which could possibly be used in a clinical trial with psoriasis patients, although for patients suffering from psoriatic arthritis, a systemic formulation might be preferable," says Simmet.

\section{Katrin Legg}

Original article Wang, $\mathrm{H}$. et al. Targeting NF- $\mathrm{KB}$ with a natural triterpenoid alleviates skin inflammation in a mouse model of psoriasis. J. Immunol. 183, 4755-4763 (2009). 